\title{
El tratamiento del hipertiroidismo con iodo radiactivo aumenta la incidencia de oftalmopatía
}

Relation between therapy for hyperthyroidism and the course of Grave's ophthalmopathy.

Marcocci C, Bogazzi F et al. N Engl J Med 1998;338:73-8

Objetivo

Evaluar los efectos del iodo (con o sin corticoides iniciales) y el metimazol en la progresión de oftalmopatía de Graves (OG) en pacientes con enfermedad de Graves.

Diseño

Ensayo randomizado, prospectivo, simple ciego. Seguimiento a un año.

Lugar

Servicio de Endocrinología y Oftalmología, Universidad de Pisa, Italia

Pacientes

Se incluyeron 450 pacientes con enfermedad de Graves con OG leve o ausente. Se excluyeron los pacientes con $0 \mathrm{G}$ severa que requirieran radioterapia orbital o corticoides, los que presentaran bocios grandes que requieran cirugía, y los que tenían contraindicaciones para recibir corticoides.

\section{Intervención}

Todos los paciente se trataron con metimazol durante 3-4 meses. Luego se randomizaron a una de las tres ramas: 1 ) Iodo radiactivo $120-$ $150 \mu$ Ci por gramo de tejido tiroideo $(n=150) ; 2)$ Iodo radiactivo + prednisona $0.4-0.5 \mathrm{mg} / \mathrm{kg} /$ día desde el día tres post iodo hasta el mes, y luego dosis decrecientes por dos meses más $(n=145)$; 3 ) Metimazol por 18 meses $(n=148)$. Siete pacientes abandonaron tempranamente el estudio y no fueron analizados. La función tiroidea se evaluó cada 1-2 meses y fue corregido el hipo o hipertiroidismo.

\section{Medición de resultados principales}

Un oftalmólogo, ciego al tratamiento instituido, examinó a los pacientes cada 1-2 meses. Evaluó la exoftalmia, y la actividad de la OG. También se documentó la percepción subjetiva de los pacientes.

\section{Resultados principales}

De los 23 pacientes tratados con iodo en los cuales se desarrolló o progresó la OG, los cambios ocurrieron en los primeros seis meses. En 8 de ellos la OG fue persistente y requirió tratamiento. La proporción de fu- madores fue mayor en el grupo en el que la OG se desarrolló o progresó comparada con el grupo en el que no hubo cambios ( $83 \%$ vs. $50 \%$, $\mathrm{p}<0.001$ ). No hubo relación entre el estado funcional tiroideo y la OG.

Evolución de la $\mathrm{OG}$ con los distintos tratamientos

\begin{tabular}{|c|c|c|c|}
\hline & Iodo $(n=150)$ & +predmisona $(n=1$ & etimazol $(n=148)$ \\
\hline $\begin{array}{l}\text { Desarrollo o progresión } \\
\text { de OG (n) \# } \\
\qquad(\% \text {, IC95\%) }\end{array}$ & $\begin{array}{c}23 \text { de } 150 \\
15 \%(10-22 \%)\end{array}$ & 0 de 145 & $\begin{array}{c}4 \text { de } 148 \\
3 \%(1-7 \%)\end{array}$ \\
\hline $\begin{array}{r}\text { Con OG previa (n) } \\
(\%, \text { IC95\%) }\end{array}$ & $\left\{\begin{array}{c}17 \text { de } 72 \\
24 \%(14-35 \%)\end{array}\right.$ & 0 de 75 & $\begin{array}{c}3 \text { de } 74 \\
4 \%\end{array}$ \\
\hline $\begin{array}{r}\text { Sin OG previa }(n) \\
(\%, \text { IC95\%) }\end{array}$ & $\left\{\begin{array}{c}6 \text { de } 78 \\
8 \%(3-16 \%)\end{array}\right.$ & 0 de 70 & $\begin{array}{c}1 \text { de } 74 \\
1 \%\end{array}$ \\
\hline $\begin{array}{l}\text { Regresión \#\# Con OG } \\
\text { previa (n) } \\
\qquad(\%, \text { IC95\%) }\end{array}$ & 0 de 72 & $\begin{array}{c}50 \text { de } 75 \\
67 \%(55-77 \%)\end{array}$ & $\begin{array}{c}3 \text { de } 74 \\
4 \%(1-11 \%)\end{array}$ \\
\hline $\begin{array}{l}\text { Sin cambios Con OG } \\
\text { previa (n) } \\
\qquad(\%, \text { IC95\%) }\end{array}$ & $\begin{array}{c}55 \mathrm{de} 72 \\
76 \%\end{array}$ & $\begin{array}{c}25 \text { de } 75 \\
33 \%\end{array}$ & $\begin{array}{c}68 \mathrm{de} 74 \\
92 \%\end{array}$ \\
\hline $\begin{array}{l}\text { Sin OG previa }(\mathrm{n}) \\
\qquad(\%, \mathrm{IC} 95 \%)\end{array}$ & $\begin{array}{c}72 \text { de } 78 \\
92 \%\end{array}$ & $\begin{array}{c}70 \text { de } 70 \\
100 \%\end{array}$ & $\begin{array}{l}63 \text { de } 74 \\
99 \%\end{array}$ \\
\hline $\begin{array}{l}\text { Tratamiento con Radio- } \\
\text { terapia o corticoides en } \\
\text { altas dosis (\%, IC05\%) }\end{array}$ & $5 \%(2-10 \%)$ & $0 \%$ & $1 \%(0-4 \%)$ \\
\hline
\end{tabular}

\# Las frecuencia de progresión fue significativamente mayor en el grupo iodo que en los otros dos $(p<0.001)$. \#\# La mejoría fue mayor en el grupo iodo+prednisona que en los otros dos $(p<0.001)$.

\section{Conclusiones}

El iodo radiactivo provocó o empeoró la $0 \mathrm{G}$ más frecuentemente que el metimazol. La OG fue en general transitoria y pudo ser prevenida con la administración de prednisona.

\section{COMENTARIO}

A pesar de que el manejo del hipertiroidismo se realiza en general junto al especialista, es muy importante para el médico de atención primaria estar al tanto de los riesgos y beneficios de las alternativas terapéuticas. Las dos estrategias más utilizadas en la enfermedad de Graves son las drogas anti-tiroideas (ej. Metimazol) y el iodo radiactivo. Ambos son igualmente efectivos para controlar el hipertiroidismo; la principal diferencia radica en los efectos adversos y la tasa de recurrencias. Al suspender el metimazol el hipertiroidismo puede recurrir, y el iodo puede provocar hipotiroidismo permanente con el tiempo. En cuanto al efecto sobre la $\mathrm{OG}$, este estudio confirma el hallazgo de otros en los que el iodo tiene un efecto deletereo (1) mientras que el metimazol no la afecta (2).
Estos resultados son más categóricos ya que los pacientes fueron seguidos frecuentemente y el desarrollo de hipotiroidismo con iodo se corrigió a la brevedad -se sabe que el aumento de TSH posttratamiento promueve la OG- (3). También confirma que los fumadores tienen mayor riesgo de $0 \mathrm{G}$. Estos resultados deben considerarse en el contexto de la historia natural de esta enfermedad. La OG es clínicamente aparente en el $50 \%$ de los pacientes con Graves; en el $60 \%$ de estos es leve, y en el restante $40 \%$ es más severa (con diplopía y pérdida de la visión). Para la aplicación clínica parece razonable que sólo los pacientes con OG importante o con factores de riesgo para desarrollarla (fumadores, aumento de TSH post-tratamiento), reciban metimazol o iodo asociado a prednisona.

Dr. Federico Augustovski

Unidad de Medicina Familiar y Preventiva. Hospital Italiano de Buenos Aires.

\footnotetext{
Referencias
1. Tallstedt L, Lundell G, Torring 0, y col. Occurrence of ophthalmopathy after treatment for Graves' hyperthyroidism. N EngL J Med 1992;326:1733-8.

1. Tallstedt L, Lundell G, Torring 0, y col. Occurrence of ophthalmopathy after treatment for Graves' hyperthyroidism.
2. Sridama V, De Groot LJ. Trreatment of Graves' disease and the course of ophthalmopathy. Am J Med 1989:87:70-3.

3. Kung AW, Yau CC, Cheng A. The incidence of ophtalmopathy after iodine therapy for Graves' hyperthyroidism: prognostic factors and the role of methimaxole. J Clin Endocrinol Metab 1994;79:542-6.

4. Perros P, Crombie AL, Kendall-Taylor P. Natural hystory of thyroid associated ophtalmopathy. Clin Endocrinol 1995:42:45-50.
} 